\title{
Repurposing cationic amphiphilic drugs as adjuvants to induce lysosomal siRNA escape in nanogel transfected cells
}

Freya Joris ${ }^{1}$, Lynn De Backer ${ }^{1}$, Thijs Van de Vyver ${ }^{1}$, Chiara Bastiancich ${ }^{2}$, Stefaan C. De Smedt ${ }^{1, *}$ and Koen Raemdonck ${ }^{1, *}$

${ }^{1}$ Lab of General Biochemistry and Physical Pharmacy, Faculty of Pharmaceutical Sciences, Ghent University, Ghent, 9000, Belgium

${ }^{2}$ Louvain Drug Research Institute, Advanced Drug Delivery and Biomaterials, Université Catholique de Louvain, Brussels, 1200, Belgium

* To whom correspondence should be addressed. Tel: +32 926480 78; Fax: +32 926481 89; Email: koen.raemdonck@ugent.be. Correspondence may also be addressed to Stefaan De Smedt. Tel:+32 926480 76; Fax: +32 926481 89; Email: stefaan.desmedt@ugent.be 


\section{ABSTRACT}

Cytosolic delivery remains a major bottleneck for siRNA therapeutics. To facilitate delivery, siRNAs are often enclosed in nanoparticles (NPs). However, upon endocytosis such NPs are mainly trafficked toward lysosomes. To avoid degradation, cytosolic release of siRNA should occur prior to fusion of endosomes with lysosomes, but current endosomal escape strategies remain inefficient. In contrast to this paradigm, we aim to exploit lysosomal accumulation by treating NP-transfected cells with low molecular weight drugs that release the siRNA from the lysosomes into the cytosol. We show that FDA-approved cationic amphiphilic drugs (CADs) significantly improved gene silencing by siRNAloaded nanogels in cancer cells through simple sequential incubation. CADs induced lysosomal phospholipidosis, leading to transient lysosomal membrane permeabilization and improved siRNA release without cytotoxicity. Of note, the lysosomes could be applied as an intracellular depot for triggered siRNA release by multiple CAD treatments.

Keywords: drug repurposing, siRNA delivery, nanogels, phospholipidosis, lysosomal membrane permeabilization, cationic amphiphilic drugs

\section{GRAPHICAL ABSTRACT}
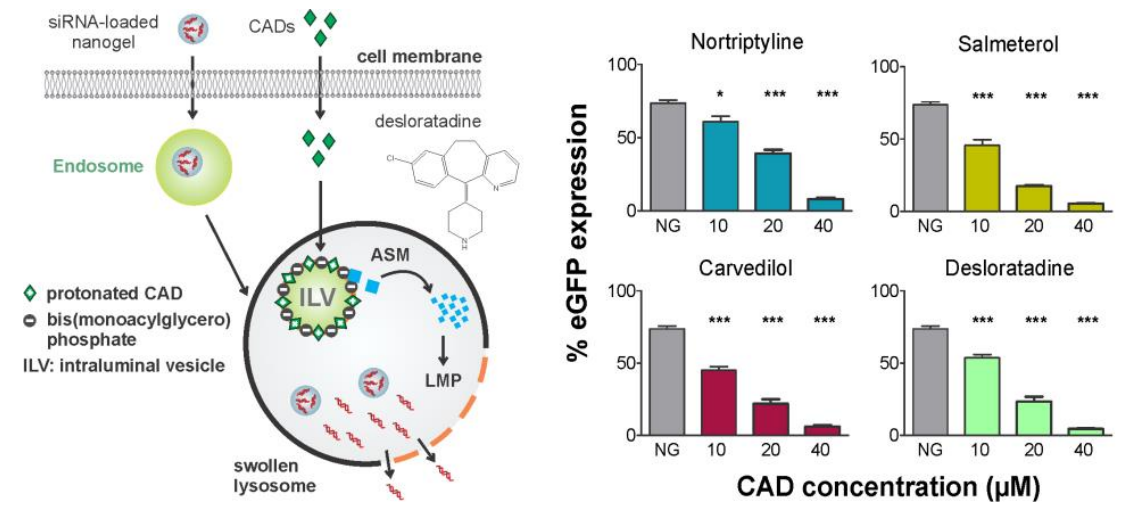

Through functional inhibition of acid sphingomyelinase (ASM), cationic amphiphilic drugs (CADs) induce non-lethal lysosomal membrane permeabilization (LMP), enhancing the cytosolic delivery of siRNA and improving target gene knockdown. 


\section{INTRODUCTION}

The cytosolic delivery of RNA interference (RNAi)-effectors, such as small interfering (si)RNA, efficiently and specifically induces post-transcriptional gene silencing [1, 2]. In theory any gene of interest can be targeted, making siRNAs promising therapeutic candidates for a myriad of diseases $[3,4]$. Since their negative charge and relatively high molecular weight hinder passive diffusion over the cell membrane, siRNA is generally enclosed in a nanocarrier to ensure cellular uptake [5]. Such carriers are internalized by cells via one or more endocytic pathways but are progressively trafficked to the lysosomes, where both particle and cargo face degradation. However, as cytosolic siRNA delivery is needed to activate the RNAi pathway and reduce gene expression, lysosomal entrapment is generally regarded as a non-functional dead end [5]. To avoid lysosomal degradation, endosomal escape should occur prior to fusion of the endosomes with the lysosomes [6, 7]. Unfortunately, the rapid trafficking towards the lysosomes strongly limits the time-window to induce release [8]. In addition, it is believed that endosomal escape strategies should target early or late endosomes since the induction of severe lysosomal membrane damage may trigger cell death, e.g. through leakage of lysosomal cathepsins [6, 9]. Despite many efforts to improve endosomal escape through optimal nanoparticle (NP) design, state-of-the-art siRNA NPs largely fail to deliver, as the bulk of the internalized siRNA dose remains entrapped in the lysosomes [10,11]. Opposed to the current paradigm, we propose to specifically exploit the lysosomes for cytosolic siRNA delivery by repurposing cationic amphiphilic drugs (CADs) as low molecular weight adjuvants. CADs are pharmacologically diverse compounds that preferentially accumulate in lysosomes given their amphiphilic and weak basic properties [12]. CADs are able to passively diffuse into the acidified lysosomal lumen where they are protonated upon which their re-diffusion into the cytosol is hindered, a phenomenon referred to as 'ion-trapping' $[12,13]$. Here, protonated CADs insert in intra-lysosomal membranes with their hydrophobic segment, causing the detachment of the membrane-associated acid sphingomyelinase (ASM) and its subsequent degradation by cathepsins in the lysosomal lumen (Figure 1) [14].

Thus, many CADs are known as functional inhibitors of ASM (FIASMAs) [12, 15]. ASM catalyzes the hydrolysis of sphingomyelin (SM) to ceramide, thereby playing an important role in the maintenance of the lipid homeostasis and lysosomal membrane integrity. Indeed, SM accumulation upon ASM inhibition is associated with induction of lysosomal membrane permeabilization (LMP) in cancer cells (Figure 1) $[14,16,17]$. Here, we aimed to evaluate if this phenomenon can be exploited to improve cytosolic siRNA delivery following NP-mediated transfection. 


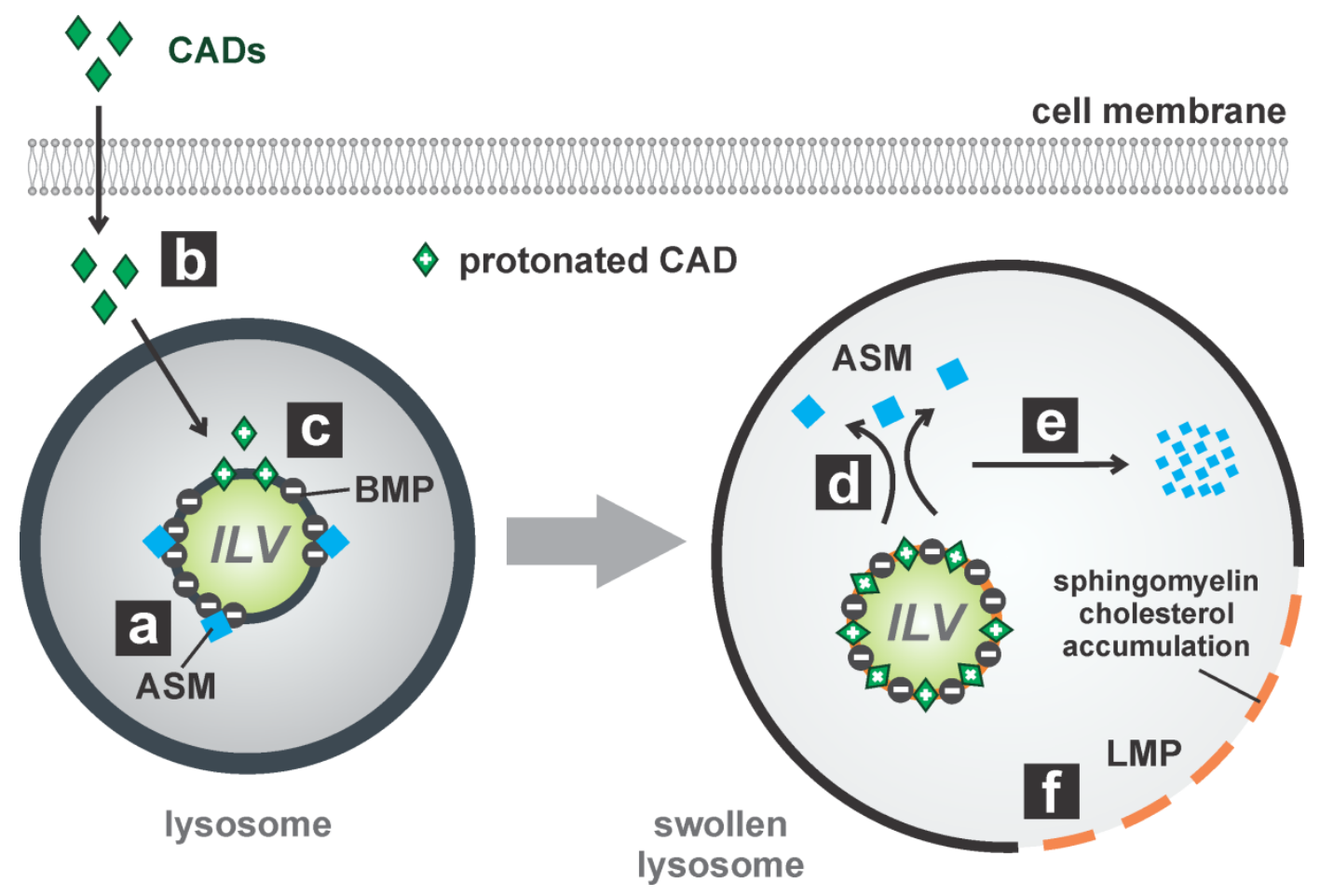

Figure 1. CAD-mediated functional ASM inhibition causes lysosomal lipid accumulation and Iysosomal membrane permeabilization. (a) Acid sphingomyelinase (ASM) is a lysosomal membrane-bound enzyme that contributes to lipid metabolism. At the acidic $\mathrm{pH}$ of the lysosomes, the cationic ASM is electrostatically bound to intraluminal vesicles (ILVs) that are enriched in the anionic lipid bis(monoacylglycero)phosphate (BMP). (b) Cationic amphiphilic drugs (CADs) preferentially accumulate in lysosomes given their amphiphilic and weak basic properties. (c) Protonated CADs insert in intra-lysosomal membranes and (d) induce ASM release into the lysosomal lumen (e) followed by degradation by cathepsins. As a result, lysosomal (phospho)lipidosis develops, accompanied by lysosomal swelling and (f) lysosomal membrane permeabilization (LMP) [14, 18].

\section{MATERIAL AND METHODS}

\section{1. siRNA duplexes}

The 21 nucleotide siRNA duplex targeting the enhanced green fluorescent protein (eGFP; siEGFP) and the control siRNA duplex (siCTRL) were purchased from Eurogentec (Belgium). This control duplex contains a unique and validated sequence that does not present any relevant homology to any known eukaryotic gene. For uptake experiments, the siCTRL duplex was labeled with a Cy5 dye at the $5^{\prime}$ end of the sense strand (Eurogentec, Belgium). Nuclease-stabilized versions of the siEGFP and SiCTRL duplexes (siSTABLE), the siGLO green transfection indicator as well as nucleasestabilized siRNA targeting polo-like kinase 1 (siPLK1) were obtained from Dharmacon (USA). The sequences and modifications of the applied siRNA duplexes are summarized in Table 1. 
Table 1. Applied siRNA sequences and duplex modifications.

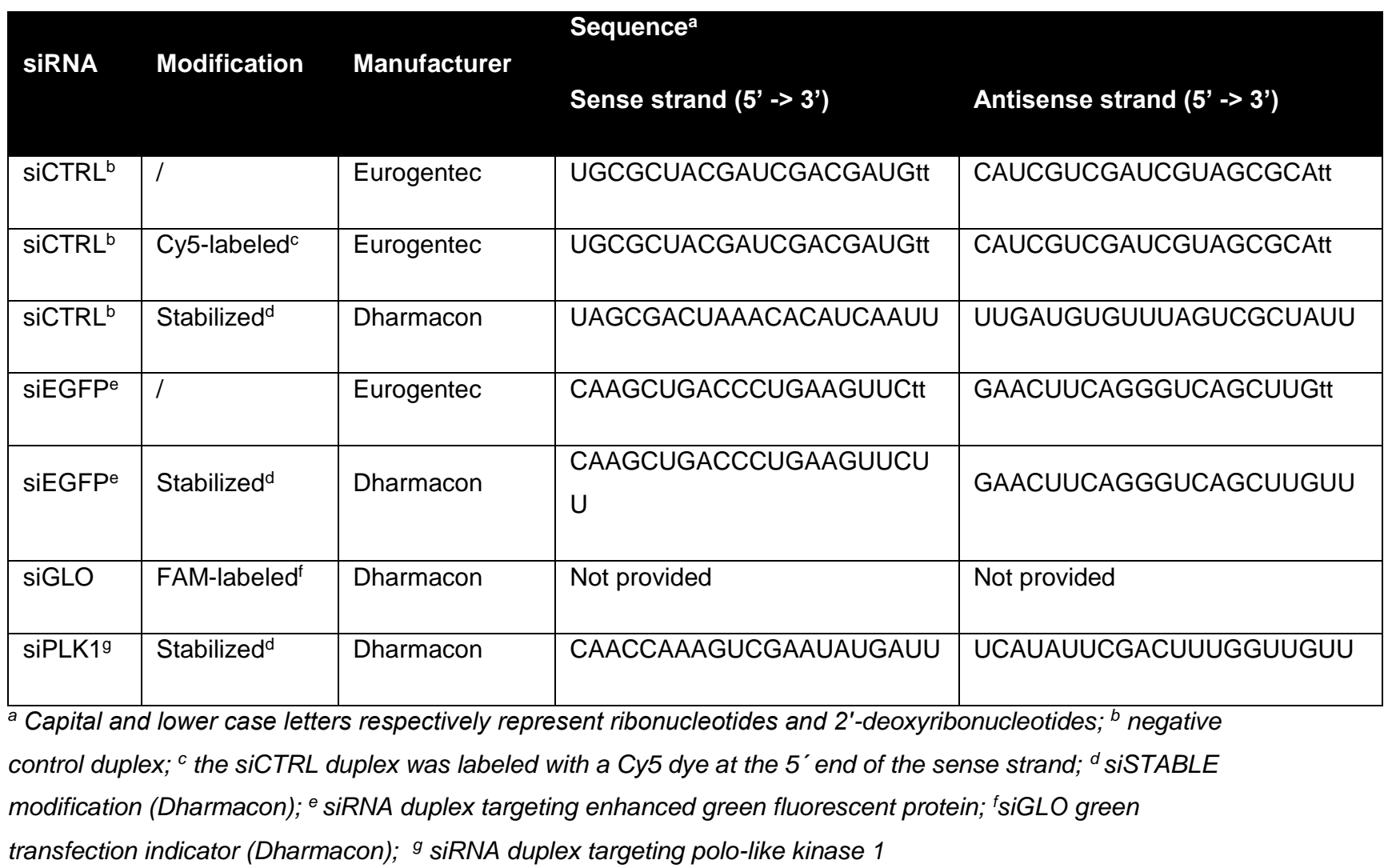

\subsection{NG complexation}

The cationic dextran nanogels (NGs) were prepared via an inverse mini-emulsion photopolymerization method [19]. For siRNA loading, a $2 \mathrm{mg} / \mathrm{mL}$ dispersion of a lyophilized NG stock was prepared in ice-cooled nuclease free water and sonicated briefly (amplitude 10\%). Subsequently, equal volumes of appropriate NG and siRNA dilutions in N-2-hydroxyethylpiperazine-N'-2ethanesulfonic acid (HEPES) buffer ( $\mathrm{pH} 7.4,20 \mathrm{mM}$ ) were mixed to a final volume of $100 \mu \mathrm{L}$ and were allowed to complex for 15 minutes at room temperature. The siRNA loaded NGs (siNGs) were subsequently diluted with HEPES $(100 \mu \mathrm{L})$ and Opti-MEM® (Invitrogen, Belgium, $800 \mu \mathrm{L}$ ) to a final volume of $1 \mathrm{~mL}$. This complexation procedure was applied for all cell-based experiments and resulted in a $30 \mu \mathrm{g} / \mathrm{mL}$ NG dispersion loaded with $2 \mathrm{nM}$ siRNA $(=0.067 \mathrm{pmol}$ siRNA $/ \mu \mathrm{g}$ NGs or $0.6 \mathrm{pmol}$ siRNA/well).

\subsection{Cell lines and cell culture conditions}

Non-small cell lung carcinoma cells (H1299), both the wild type (H1299-WT) and the eGFPexpressing variant (H1299-eGFP), were maintained in RPMI 1640 culture medium supplemented with $10 \%$ fetal bovine serum (FBS, Hyclone ${ }^{\mathrm{TM}}$, Thermo Fisher Scientific, Belgium), $2 \mathrm{mM}$ L-Glutamine and $100 \mathrm{U} / \mathrm{mL}$ penicillin/streptomycin. The cell lines were cultured in a humidified atmosphere containing $5 \% \mathrm{CO}_{2}$ at $37{ }^{\circ} \mathrm{C}$ and culture medium was renewed every other day unless the $80 \%$ confluence level 
was reached. In this case, the cells were split using $0.25 \%$ trypsin-EDTA. All products were purchased from Invitrogen (Belgium) unless specifically mentioned otherwise.

\section{4. siNG transfection and sequential adjuvant treatment}

Cells were transfected with siNGs during 4 hours at $37^{\circ} \mathrm{C}$ in a humidified atmosphere containing $5 \%$ $\mathrm{CO}_{2}$. Subsequently, the siNG dispersion was removed, the cells were washed once with phosphate buffered saline (PBS, Invitrogen, Belgium) and received fresh cell medium. In case of CAD treatment, complete cell medium containing the CAD or combination of CADs at the indicated concentrations was applied on the cells for 20 hours, unless specifically mentioned otherwise. Four different CADs were tested, namely carvedilol (CD), desloratadine (DL), nortriptyline (NT) and salmeterol (ST) (Figure 2). In additional experiments, we applied complete cell medium containing 10, 20 or $40 \mu \mathrm{M}$ chloroquine (CLQ), $30 \mu \mathrm{M}$ U18666A or $300 \mu \mathrm{M}$ 2-hydroxy oleic acid (2OHOA), the latter either alone or in combination with $10 \mu \mathrm{M}$ DL. Figure $S 2$ shows the molecular structure, $\log P$ and pKa values of the additionally applied endosomolytic adjuvants. All small molecules were obtained from SigmaAldrich (Belgium) and the stock solutions were prepared in sterile-filtered BioPerformance Certified dimethyl sulfoxide (DMSO, Sigma-Aldrich, Belgium). The final DMSO concentration on the cells did not exceed 0.16 vol\%. Following adjuvant incubation, the small molecule-containing cell medium was removed and cells were kept in $1 \mathrm{~mL}$ fresh cell medium for an additional 24 hours until analysis. It was verified that the solvent in which the CADs were dissolved (DMSO) did not influence the siNGmediated gene silencing (data not shown).

\subsection{Quantification of the siNG transfection efficiency}

For silencing experiments, H1299-eGFP cells were seeded in 24 well plates at a density of 35000 cells/well and were allowed to settle overnight. Subsequently, the cells were transfected with $0.6 \mathrm{pmol}$ siRNA/well (4 hours incubation, $300 \mu \mathrm{L}$ siNG dispersion) and treated with $0.5 \mathrm{~mL}$ cell medium containing a small molecular adjuvant ( 20 hours incubation unless specifically stated otherwise). Note that for every siEGFP or siPLK1 condition an SiCTRL sample was included to account for potential off-target effects. SiNG-mediated eGFP silencing was quantified by flow cytometry. Sample preparation consisted of washing the cells with PBS, followed by detachment with $0.25 \%$ trypsinEDTA. Next, the cells were collected, centrifuged 5 minutes at $300 \mathrm{~g}$, resuspended in $300 \mu \mathrm{L}$ flow buffer (1\% FBS in PBS) and kept on ice until analysis. For each sample the forward and side scatter as well as the green fluorescent signal were acquired for at least 15000 cells. The samples were excited with the $488 \mathrm{~nm}$ laser line and the signal was detected with the 530/30 filter using the FACSCalibur ${ }^{\mathrm{TM}}$ flow cytometer (BD Biosciences, Belgium) and BD CellQuest ${ }^{\mathrm{TM}}$ acquisition software. Finally, data analysis was performed using the FlowJo software (Tree Star Inc.) and the calculated percentage eGFP expression is presented as the mean \pm standard error of the mean (SEM) for 4 independent repeats. When probing the kinetics of the eGFP expression over an extended period of time, the eGFP signal was measured daily. Hereto, treated cells were passaged every other day and reseeded in new 24 well plates during sample preparation for the flow cytometry measurements. The 
silencing potential of the siPLK1-loaded NGs was established through evaluating the cell viability with the CellTiter $\mathrm{GLO}^{\circledR}$ assay (Promega, Belgium). Before initiating the assay, the culture plates and reconstituted assay buffer were placed at room temperature for 30 minutes. Next, the culture medium was replaced by $250 \mu \mathrm{L}$ fresh cell medium and an equal volume of assay buffer was added. To induce complete cell lysis, the plates were shaken during 2 minutes and the signal was allowed to stabilize the following 10 minutes. $100 \mu \mathrm{L}$ from each well was subsequently transferred to an opaque 96-well plate, which was measured with a GloMax ${ }^{\circledR} 96$ Microplate Luminometer (Promega, Belgium). Three independent repeats were performed and data are expressed as the mean cell viability $(\%) \pm$ SEM.

\subsection{Cell viability}

The H1299-eGFP cells were seeded, transfected with siCTRL-loaded NGs and treated with the CADs similar to the silencing experiments. Again, the CellTiter $\mathrm{GLO}^{\circledR}$ assay was performed as described above in 3 independent repeats (data are expressed as the mean \pm SEM).

\subsection{Cellular release of FITC-dextrans and Cy5-oligonucleotides}

H1299-WT cells were seeded at 105000 cells/dish in $35 \mathrm{~mm}$ diameter glass bottom microscopy dishes (Greiner Bio-One GmbH, Germany) and were allowed to settle overnight. To visualize the FITC-dextran (FD) release from lysosomes, a $1 \mathrm{mg} / \mathrm{mL}$ dispersion of $10 \mathrm{kDa}$ FD (Sigma-Aldrich, Belgium) in complete cell medium was added during 1 hour at $37^{\circ} \mathrm{C}$ in a humidified atmosphere containing $5 \% \mathrm{CO}_{2}$. Fluorescently labeled dextrans $(10 \mathrm{kDa})$ are internalized by cells through fluidphase pinocytosis and are useful probes to assess lysosomal integrity [20, 21]. To assess oligonucleotide (ON) escape, the NGs were first loaded with $100 \mathrm{nM}$ Alexa Fluor 647-labelled ON (Eurogentec, Belgium) according to the procedure described for siRNA complexation and $900 \mu \mathrm{L}$ of the ON-loaded NG dispersion was added to each dish. Following 4 hours of ON-loaded NG transfection or 1 hour incubation with FD, the cells were washed with PBS and received $1.5 \mathrm{~mL}$ fresh cell medium with or without 10,20 or $40 \mu \mathrm{M} \mathrm{DL}$. After an additional incubation period of 20 hours, the cell medium was removed and nuclei were labeled with Hoechst (Molecular Probes ${ }^{\mathrm{TM}}$, Belgium) in cell medium during 15 minutes at $37^{\circ} \mathrm{C}$. Finally, the Hoechst solution was removed, fresh cell medium was added and cells were kept at $37^{\circ} \mathrm{C}$ in a humidified atmosphere containing $5 \% \mathrm{CO}_{2}$ until imaging using a laser scanning confocal microscope (LSCM, C1si, Nikon) and a 60x oil immersion Plan Apo objective (Nikon, Belgium). The $408 \mathrm{~nm}, 488 \mathrm{~nm}$ and $633 \mathrm{~nm}$ laser lines were applied to respectively excite the Hoechst labeled nuclei, the FD and ON. During data analysis with ImageJ, both the total cell number and number of cells with a diffuse FD labeling or ON-positive nuclei were counted. Data are represented as the percentage of cells with a diffuse FD signal for minimum 225 cells per condition in 10 images and the percentage of cells with ON-positive nuclei for at least 180 cells in 10 images. FD- or ON-treated cells not exposed to DL were employed as the negative control. 


\subsection{Quantification of lysosomal volume using flow cytometry}

H1299-eGFP cells were seeded, transfected and treated with the CADs as described for the cell viability experiments. Following 20 hours of CAD treatment, the lysosomes were labeled with the LysoTracker® Deep Red (LDR) probe (Molecular Probes ${ }^{\mathrm{TM}}$, Belgium) through incubation with $1 \mathrm{~mL}$ $75 \mathrm{nM}$ LDR in cell medium for 30 minutes at $37^{\circ} \mathrm{C}$. Further sample preparations were carried out as previously described for the silencing experiments. Using the FACSCalibur ${ }^{\mathrm{TM}}$ flow cytometer and BD CellQuest $^{\mathrm{TM}}$ acquisition software, the LDR signal was detected with the 661/16 filter following excitation with the $635 \mathrm{~nm}$ laser line for at least 15000 cells per sample. Experiments were performed in biological triplicate and fold changes in LDR signal intensity are expressed as the mean \pm SEM.

\subsection{Visualizing lysosomes, phospholipids, cholesterol and sphingomyelin}

H1299-WT cells were seeded as specified for the FD release experiment and transfected with siNGs followed by a 20 hour DL treatment. Subsequently, lysosomes were labeled with LysoTracker ${ }^{\circledR}$ Red DND-99 (Molecular Probes ${ }^{\mathrm{TM}}$, Belgium). Phospholipids were stained with the LipidTOX ${ }^{\mathrm{TM}}$ red phospholipidosis detection reagent (Thermo Fisher Scientific, USA). Cholesterol could be detected following labeling with filipin. Finally, lysenin (Sigma-Aldrich, Belgium) was applied to label sphingomyelin. Detailed labeling, imaging and analysis procedures are provided in the supporting information.

\subsection{Transfection with siGLO green transfection indicator}

H1299-WT cells were seeded as described previously. For this experiment, NGs were complexed with $100 \mathrm{nM}$ green fluorescent siRNA (siGLO, Dharmacon, USA). Following transfection and $40 \mu \mathrm{M} \mathrm{DL}$ treatment, the cells were fixed with 4\% PFA and stored in Vectashield containing DAPI. The samples were imaged with a LSCM and a 100x oil immersion objective following excitation with the $408 \mathrm{~nm}$ and $488 \mathrm{~nm}$ laser line to visualize the DAPI and siGLO signal, respectively.

\subsection{Statistical analysis}

Statistical analysis was performed using the $6^{\text {th }}$ version of the GraphPad Prism software. One-way ANOVA combined with the post-hoc Dunnett test was applied to compare multiple conditions, whereas the student $t$-test was used for direct comparison of 2 conditions.

\section{RESULTS AND DISCUSSION}

\subsection{CAD treatment enhances siRNA-mediated gene silencing of nanogels}

We selected four structurally diverse, FDA-approved CADs with a distinct pharmacological activity, namely carvedilol (CD, a $\beta$-blocker), desloratadine ( $\mathrm{DL}$, an antihistamine), nortriptyline ( $N T$, an antidepressant) and salmeterol (ST, a $\beta 2$-agonist) (Figure 2). All compounds have an estimated logP $>3$ and pKa > 7.4 and can thus be classified as lysosomotropic drugs, CADs and FIASMAs [12, 15]. 


\section{CARVEDILOL DESLORATADINE NORTRIPTYLINE SALMETEROL}

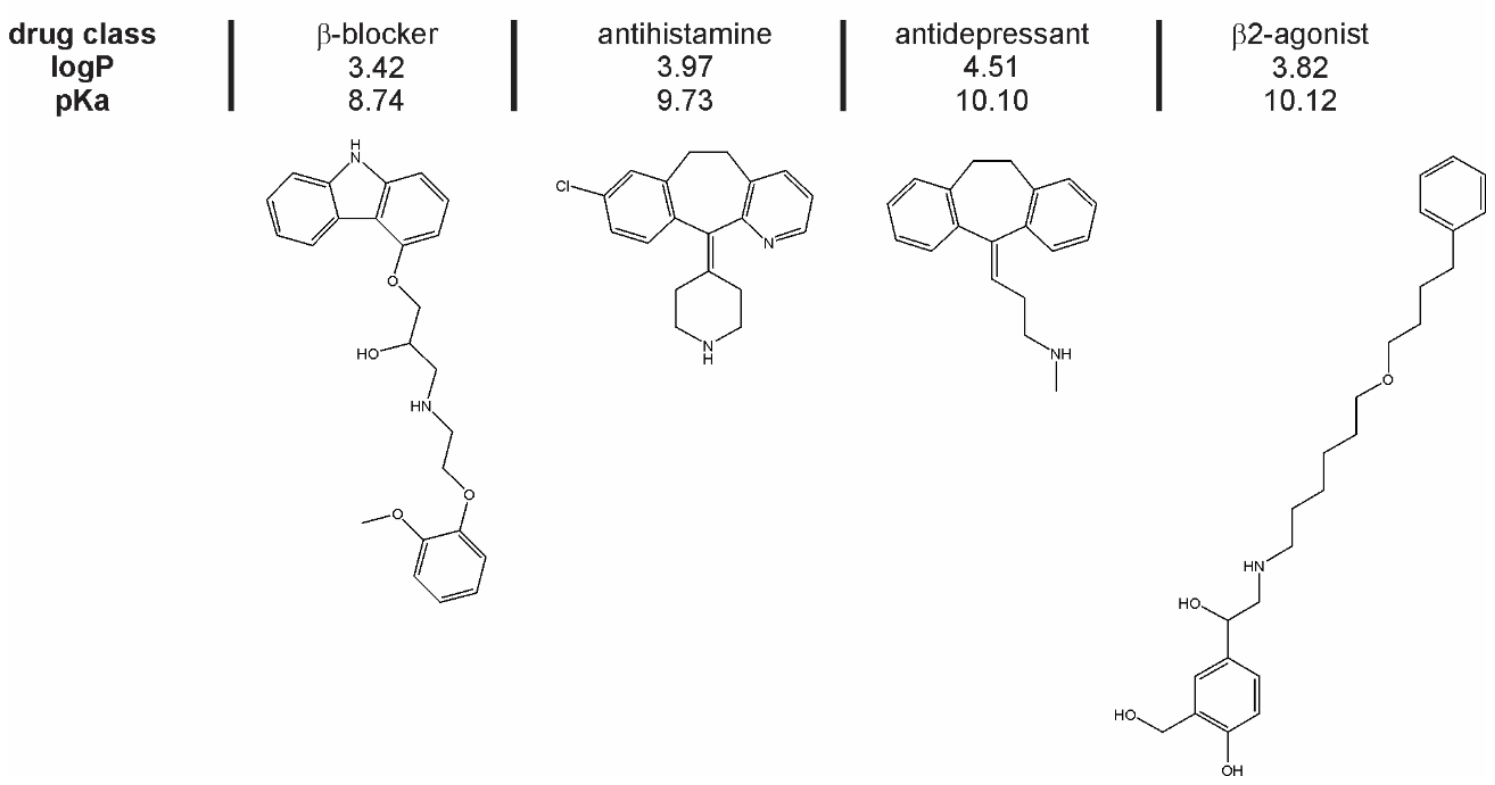

Figure 2. Applied cationic amphiphilic drugs. The drug class, logP, pKa values and molecular structure of carvedilol (CD), desloratadine (DL), nortriptyline (NT) and salmeterol (ST) [22].

As a model NP, we applied siRNA-loaded, biodegradable cationic dextran nanogels (siNGs) that have demonstrated high uptake and gene silencing efficiency in cancer cells [19, 23]. Here, non-small cell lung cancer cells (H1299) that stably express the enhanced green fluorescent protein (eGFP) were first transfected with siNGs, loaded with a suboptimal siRNA dose (2 nM or 0.6 pmol siRNA/well). In line with earlier observations, the siNGs were efficiently internalized by the $\mathrm{H} 1299$ cells (Figure S1A) and induced $25 \%$ eGFP silencing under the given experimental conditions (Figure 3A) [19, 23]. Notably, a sequential 20 hour treatment with each compound significantly improved the siNG silencing potential in a similar concentration-dependent fashion (Figure 3A, Figure S1B), despite the marked variation in drug class and molecular structure [22]. Interestingly, combining two different CADs allowed additive effects on eGFP knockdown, as shown for DL and NT (Figure 3B), which is in line with the reported additive ASM inhibition [12]. Our adjuvant approach furthermore outperformed the gold standard for siRNA transfections, i.e. Lipofectamine ${ }^{\circledR}$ RNAiMAX, when used at a similar siRNA concentration as the suboptimal siNGs ( $0.6 \mathrm{pmol}$ siRNA/well) (Figure 3C). Note that, as compared to Lipofectamine ${ }^{\circledR}$ RNAiMAX, the same level of knockdown can be achieved with a 10-fold lower dose of siRNA by sequential CAD addition to siNG-transfected cells. Such a dose sparing strategy could strongly reduce the risk of siRNA-related adverse effects. In turn, not all CADs are active in the same dose range as 10 -fold higher concentrations of dextromethorphan were required to obtain a similar adjuvant effect relative to the other CADs tested (Figure 3D). This may be attributed to steric hindrance of the basic amine reducing efficient ASM membrane detachment [22, 24]. Finally, sequential addition of the well-known endosomal escape enhancer chloroquine (CLQ) equally improved siNG-mediated silencing (Figure 3E). However, the use of CLQ as a delivery enhancer is 
limited by its systemic toxicity, while other CADs, e.g. antihistamines, may provide a safer alternative [25].

We subsequently evaluated the impact of the $C A D$ treatment, alone or sequential to the siNG transfection, on cell viability. Figure $4 A-B$ indicates that the CADs were overall well tolerated in the applied concentration range with $\mathrm{DL}$ and $\mathrm{CD}$ being the least and most cytotoxic, respectively.

\subsection{Desloratadine improves the cytosolic delivery of macromolecules}

We hypothesized that the sequential CAD treatment improved the siNG silencing potential by facilitating siRNA release into the cytosol, through the induction of lysosomal membrane permeabilization (LMP). To visualize LMP, the H1299 lysosomes were first labeled with FITC-dextran (FD, $10 \mathrm{kDa}$ ). For dextrans of this molecular weight it is known that upon fluid phase uptake they are efficiently trafficked towards the lysosomal compartments, making them ideal probes to monitor lysosomal integrity. The cytosolic release of labeled dextrans is a frequently applied method to probe LMP $[20,21]$. Upon lysosomal leakage of FD due to LMP, the affected cells should show a diffusely stained cytosol in contrast to the typical punctate pattern indicative of lysosomal sequestration [20]. As expected, the punctate pattern was observed in the bulk of the untreated cells (Figure 4C). Upon $\mathrm{DL}$ adjuvant treatment, we observed a concentration-dependent increase in the percentage of cells with a diffuse FD labeling, reaching up to $26 \%$ with $40 \mu \mathrm{M}$ DL. Comparable results were obtained by applying NGs loaded with negatively charged Cy5-labeled oligonucleotides (Cy5-ON) (data not shown) [26]. Although the percentage of positive cells correlates well for both methods, it was lower than anticipated based on the eGFP gene silencing results. Indeed, upon cytosolic release of a limited amount of FD or Cy5-ON, the labels are diluted in the cell and might fall below the detection limit of a standard confocal microscope [11].

\subsection{The lysosomal compartment is affected by CAD treatment}

Next, we assessed the impact of the CAD treatment on the lysosomes of siNG-transfected cells by quantifying the total endo-lysosomal volume with the lysosomotropic dye LysoTracker ${ }^{\circledast}$ Deep Red (LDR). Figure 5A-B shows that all four CADs evoked a similar concentration-dependent signal increase compared to untreated and siNG transfected cells. These observations could visually be confirmed by confocal microscopy, showing a marked enlargement of LysoTracker ${ }^{\circledR}$ Red labeled vesicles (Figure 5C), as also reflected in the LysoTracker ${ }^{\circledR}$ Red signal area (Figure 5D). These data corroborate earlier findings on lysosomal volume expansion in cells that had been exposed to CADs [27]. 

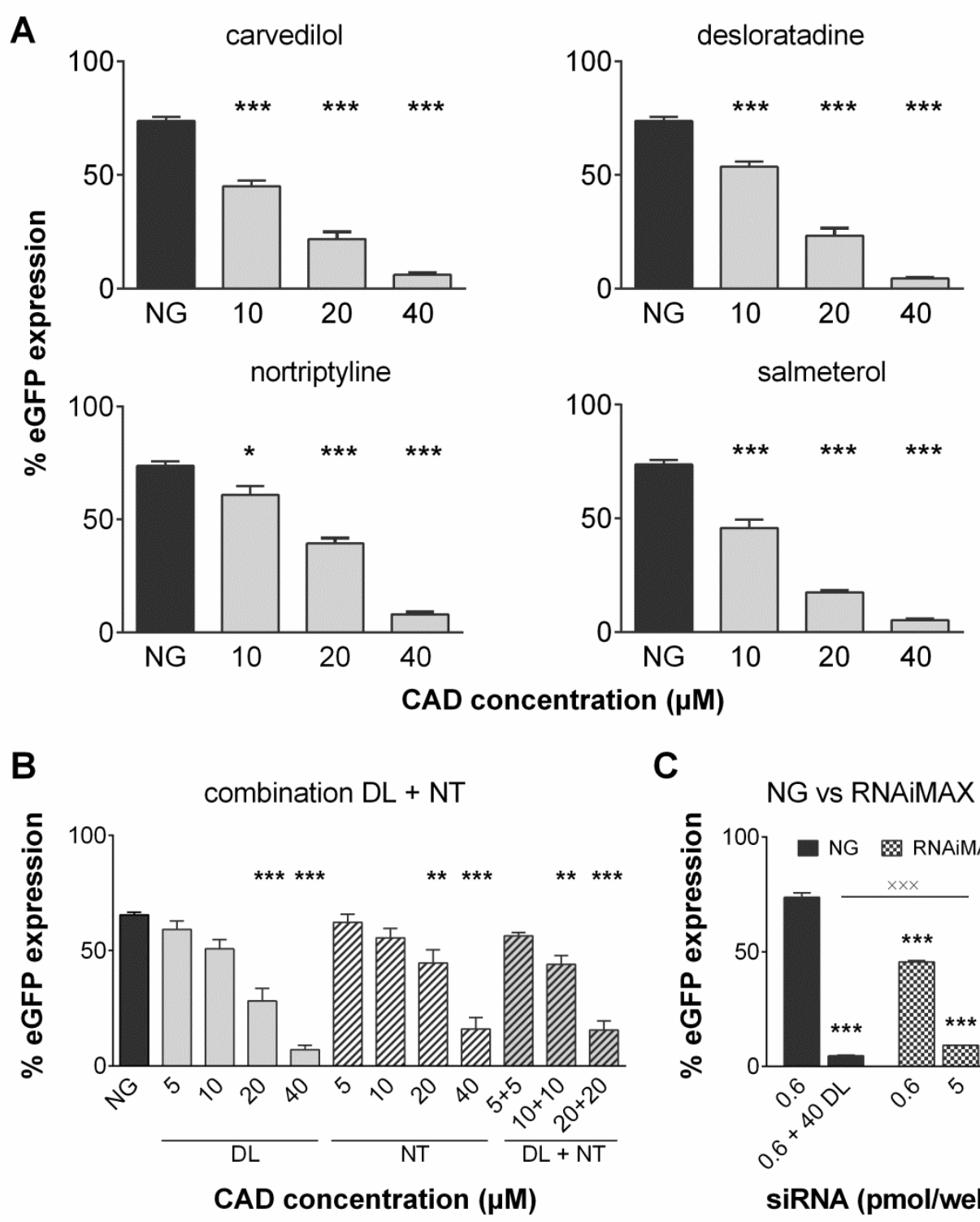

C NG vs RNAiMAX
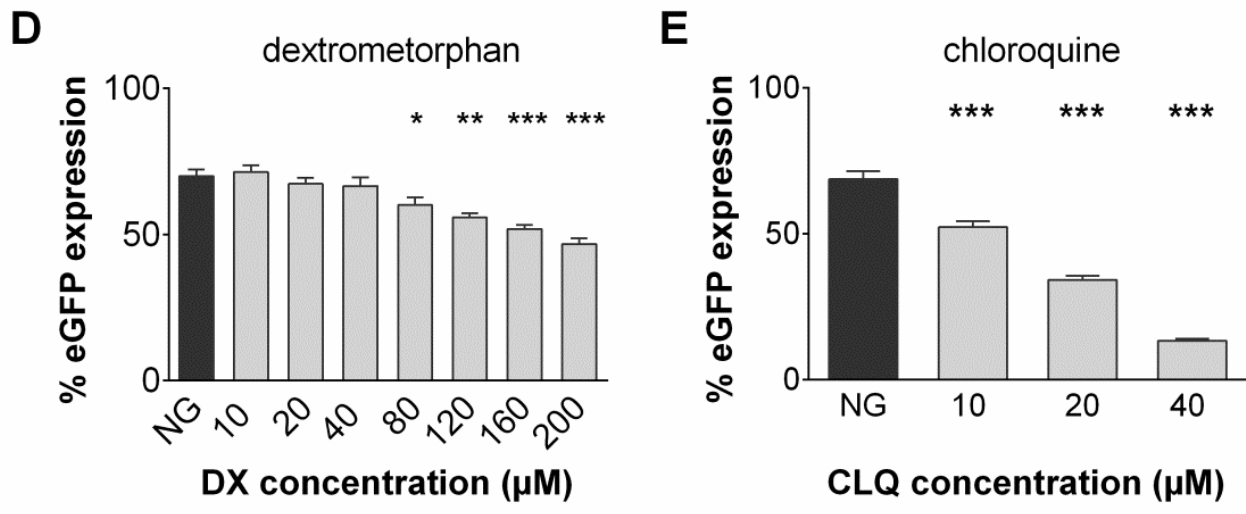

Figure 3. CADs boost siRNA-mediated gene silencing upon siNG transfection. (A) Sequential treatment of siNG-transfected H1299-eGFP cells with CADs during 20 hours improved eGFP silencing in a concentration-dependent manner. (B) Combinations of desloratadine $(D L)$ and nortriptyline (NT) evoked additive effects on eGFP silencing. (C) Comparison of eGFP knockdown 
between siNGs and Lipofectamine ${ }^{\circledR}$ RNAiMAX. (D) Impact of sequential addition of dextrometorphan (DX) on siNG-mediated eGFP silencing in H1299-eGFP cells. (E) The endosomal escape enhancer chloroquine (CLQ) equally improves siNG-mediated gene silencing. Data are represented as mean \pm SEM for minimum 3 independent repeats $(n=3)$. Statistical significance, with respect to $N G$ transfection alone, is indicated when appropriate $\left({ }^{*} p<0.05,{ }^{* *} p<0.01,{ }^{* * *} p<0.005\right)$. Statistical significance between $N G+40 \mu M D L$ and RNAiMAX is indicated when appropriate $\left.{ }^{\left({ }^{x x}\right.} p<0.005\right)$.

A

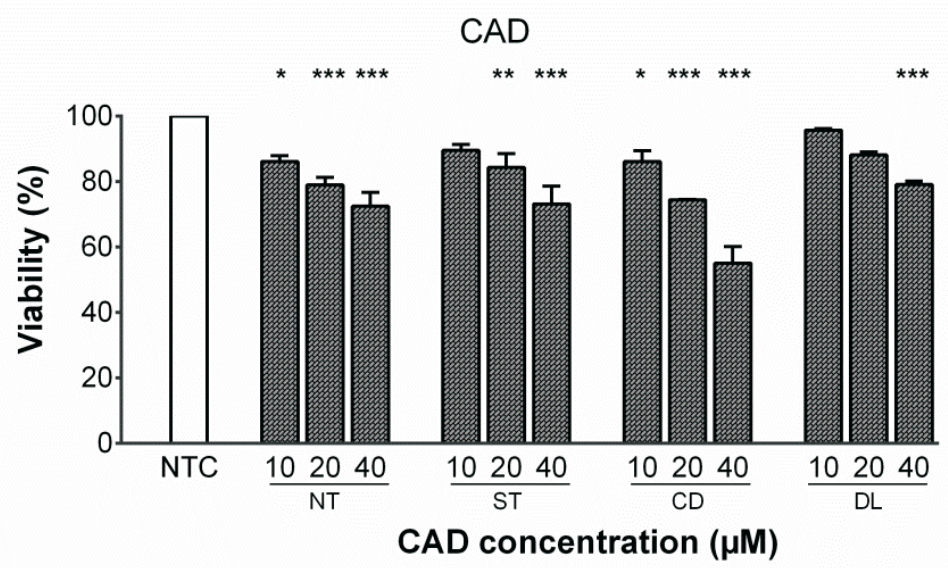

B

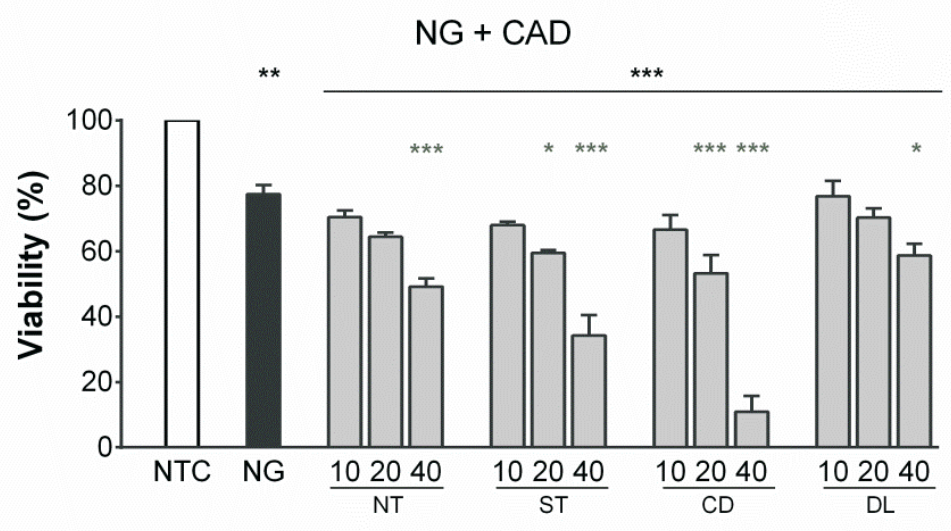

C

CAD concentration $(\mu \mathrm{M})$

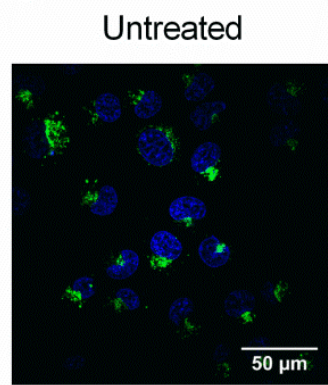

$2.3 \%$
$10 \mu \mathrm{M} \mathrm{DL}$

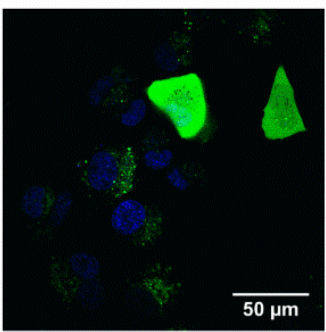

$12.9 \%$
$20 \mu \mathrm{M} \mathrm{DL}$

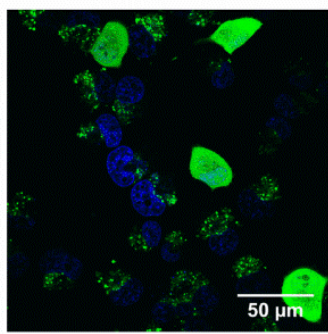

$16.5 \%$
$40 \mu \mathrm{M} \mathrm{DL}$

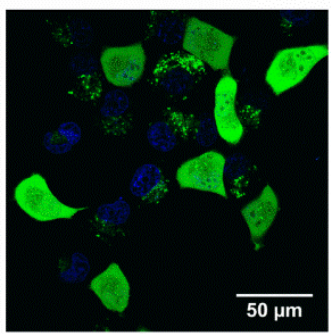

$26.3 \%$

Figure 4. CADs improve macromolecule release to the cytosol while inducing limited cytotoxicity levels. Cell viability of H1299-eGFP cells following CAD treatment alone $(A)$ or sequential $N G$ transfection and CAD addition $(B)$. Data reflect the mean $\pm S E M$ ( $n=3$ independent repeats) and statistical significance is indicated when appropriate $\left({ }^{*} p<0.05,{ }^{* *} p<0.01,{ }^{* * *} p<0.005\right)$. 
In (B) the black * represent significant variations relative to the untreated control (NTC), whereas the gray * resemble significant differences with respect to the cells transfected with siCTRL-NGs. (C) Representative confocal images from the intracellular FITC-dextran (10 kDa) distribution in untreated H1299-WT cells or cells incubated with 10, 20 or $40 \mu M$ DL following FITC-dextran uptake via endocytosis. The values below the images correspond to the percentage of cells with a diffuse FITCdextran labeling. The scale bar corresponds to $50 \mu \mathrm{m}$. (NTC = not treated control, $N G=$ nanogels, $C A D=$ cationic amphiphilic drug, $N T=$ nortriptyline, $S T=$ salmeterol, $C D=$ carvedilol, $D L=$ desloratadine)

Interestingly, this CAD-induced lysosomal swelling furthermore increased the cellular granularity, as indicated by the augmented side scatter (SSC) signal (Figure 5E-F, Figure S3A) and the combination of CADs resulted in additive SSC increases (Figure S3B). In line with the concentration-dependent effect of dextromethorphan on siNG-induced gene silencing (Figure 3D), only doses above $80 \mu \mathrm{M}$ significantly augmented the SSC signal (Figure S3C), although the effect was less outspoken compared to the other molecules tested.

\subsection{CADs induce a lysosomal storage disease-like phenotype}

CADs are known lysosomotropic agents and phospholipidosis (PLD) inducers, although the underlying cause is still unclear [28]. Labeling CAD-treated cells with the PLD detection reagent LipidTOX ${ }^{\mathrm{TM}}$ Red (Figure 6A), revealed that DL treatment induced PLD. The lipids accumulated in vesicular structures, which enlarged as a function of the DL concentration. Again, only higher doses of dextromethorphan $(\geq 80 \mu \mathrm{M})$ clearly induced vesicular phospholipid accumulation.

Such lysosomal swelling due to lipid accumulation is a general phenotypical feature of lysosomal storage disorders [29]. CADs are known to induce a Niemann-Pick disease (NPD) phenotype [30]. NPD is a lysosomal storage disorder caused by either a depletion of the Niemann-Pick type C1 (NPC1) protein that is involved in cholesterol transport (NPD type C) or a genetic defect in the ASM enzyme (NPD type A). Both NPD type A and C present a similar phenotype that is characterized by less stable, enlarged lysosomes due to the accumulation of cholesterol and sphingolipids, including SM [24, 31]. Interestingly, sequential treatment of siNG transfected cells with U18666A, a structurally different low molecular weight NPD inducer (Figure S2), also markedly increased cellular granularity (Figure 6B) as well as siNG-mediated gene silencing (Figure 6C). 
A

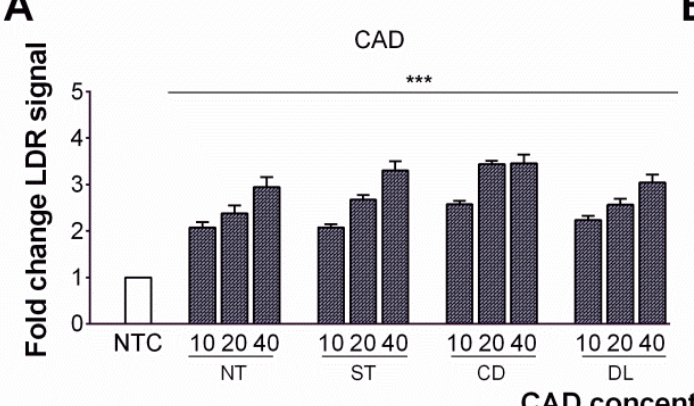

C
B

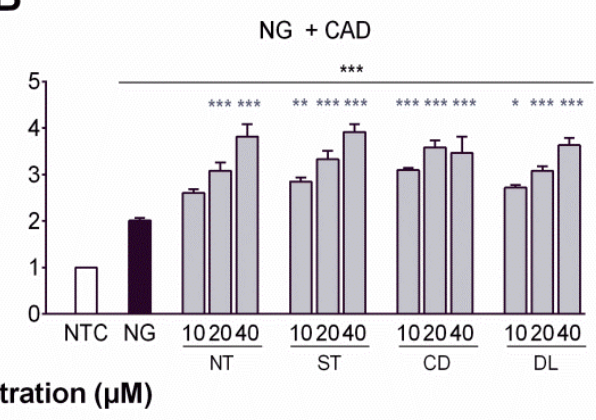

NG

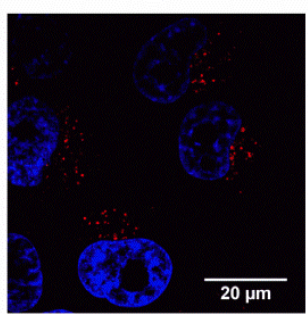

$\mathrm{NG}+20 \mu \mathrm{M} \mathrm{DL}$

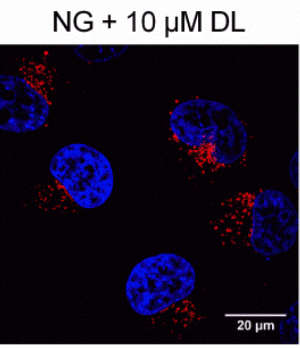

D

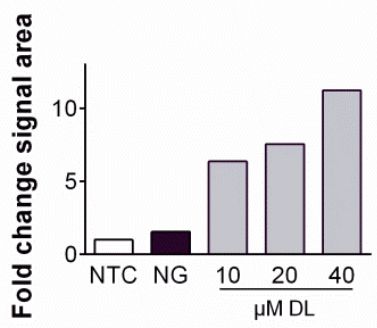

$\mathrm{NG}+40 \mu \mathrm{M} \mathrm{DL}$
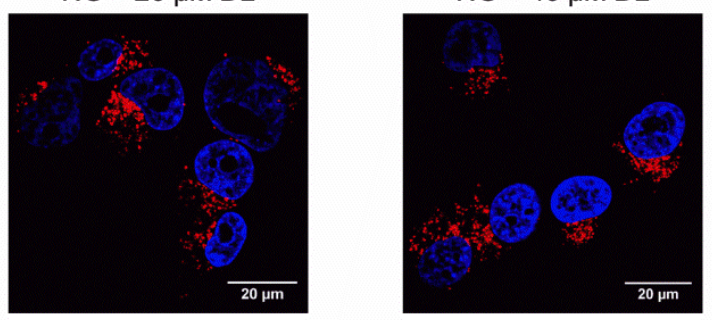

E

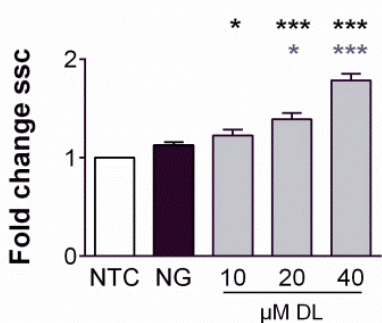

F
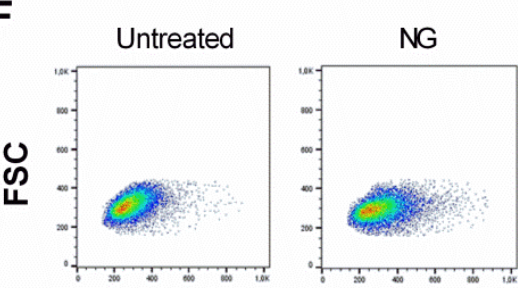
$\mathrm{NG}+10 \mu \mathrm{M} \mathrm{DL}$
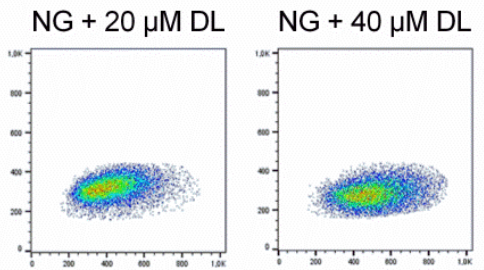

SSC

Figure 5. CADs affect the lysosomal compartment. Fold change in LDR signal measured via flow cytometry for H1299-eGFP cells treated only with the CADs (A) or the combination of siNGs and CADs (B). (C) Representative confocal images showing the lysosomal compartment (red) following LysoTracker ${ }^{\circledR}$ Red labeling for untreated H1299-WT cells, NG-transfected cells or cells transfected with siNGs followed by 10, 20 or $40 \mu M D L$ treatment. (D) Fold increase in signal area relative to the untreated control quantified from the confocal images in (C). (E) Fold change in side scatter induced 
by NG transfection or sequential treatment with $D L$. (F) Representative scatter plots for untreated cells, NG-transfected cells or cells transfected with siNGs followed by 10, 20 or $40 \mu M D L$ treatment. Data are represented as the mean $\pm \operatorname{SEM}(n=3)$ and statistical significance is indicated when appropriate, in black when referring to the untreated control and in gray when compared to NG transfected cells $\left({ }^{*} p<0.05,{ }^{* *} p<0.01,{ }^{* * *} p<0.005\right) .\left(N T C=\right.$ not treated control, LDR $=$ LysoTracker $^{\circledR}$ Deep Red, $C A D=$ cationic amphiphilic drug, $N G=$ nanogels, $N T=$ nortriptyline, $S T=$ salmeterol, $C D$ = carvedilol, $D L=$ desloratadine, $F S C=$ forward scatter, $S S C=$ side scatter)

In line with our results on lysosomal swelling (Figure 5C) and phospholipid accumulation, we observed vesicular accumulation of cholesterol and SM upon CAD treatment (Figure 6D-E, Figure S4), further supporting the notion that CADs reduce ASM activity and induce a NPD phenotype. The additive effect of 2-hydroxy oleic acid (2-OHOA), a known activator of the SM synthase, and DL on the siRNA-mediated gene silencing indeed suggests an involvement of the disturbed SM homeostasis in the improved cytosolic siRNA delivery (Figure 6F) [32]. Of note, recent literature reports have shown enhanced siRNA delivery in NPC1- cells and upon NPC1 inhibition by NP3.47, a putative inhibitor of the NPC1 protein that was initially discovered in a compound screen to identify blockers of Ebola viral infection. These results were however explained by reduced exocytosis of the lipid-based NP as NPC1 is recognized as a key player in cellular recycling [8, 33]. Likewise, it cannot be excluded that reduced recycling of the siNGs contributed to the CAD-induced adjuvant effect since ASM inhibition is also reported to inhibit vesicle fusion [31]. Altogether, these results suggest that the acquired PLD/NPD phenotype as a result of CAD incubation is responsible for the observed enhancement of siRNA delivery.

\subsection{The impact of desloratadine incubation time and multiple treatments}

All experiments described above involved a 20 hour CAD treatment. Interestingly, Figure 7A shows that both a 2 hour and 20 hour incubation improved siNG-mediated gene silencing to a similar extent. Of note, the SSC signal was significantly lower in cells exposed 2 hours to DL as compared to 20 hours (Figure 7B), which suggests that the increased cellular granularity is only transiently induced. Importantly, a 2 hour DL incubation period did not cause any additional cytotoxicity compared to the siNGs alone (Figure 7C). These results imply that CADs only trigger minor and transient LMP, sufficient to allow improved siRNA release in the cytosol while avoiding lysosomal cell death. This non-lethal LMP furthermore suggests that small pores are created in the lysosomal membrane, which solely allows uncomplexed siRNA to transfer to the cytosol. Our data corroborate the recent finding that sub-lethal concentrations of the apoptotic L-leucyl-L-leucin-methyl ester (LLOMe) transiently permeabilize lysosomal membranes followed by fast restoration of lysosomal integrity. Moreover, the LLOMe-induced LMP only allowed cytosolic leakage of macromolecules below $10 \mathrm{kDa}$ but not the larger apoptosis-evoking cathepsins (20-30 kDa) [34]. Extensive LMP entails the release of lysosomal cathepsins as well as cytosolic acidification, leading to uncontrolled cell death. On the other hand, also partial LMP might evoke lysosomal cell death in the absence of generalized cytoplasmic acidification through the release of cathepsin $B$ and $D$, which maintain activity at neutral $\mathrm{pH}$. However, 
in the latter case the action of endogenous cathepsin inhibitors can safeguard cell survival, which could in part also explain the lack of cell death upon controlled CAD exposure in our experiments $[9$, $35,36]$. Of note, the delivery efficiency of siNGs loaded with the larger 25-27mer Dicer-substrate siRNAs (DsiRNA, 18kDa) could also be promoted by sequential CAD incubation (Figure S5), in contrast to eGFP-encoding mRNA ( 500kDa; Figure S6), implying that higher adjuvant concentrations and more extensive LMP might be required for cytosolic delivery of larger nucleic acid therapeutics. However, this assumption requires further experimental validation.

Next, we investigated whether DL treatment influenced the kinetics of the siNG-mediated gene silencing. A DL concentration-dependent improvement of initial gene silencing was obtained in correspondence to previous results (Figure 7D). From day 2 onwards, the relative eGFP expression steadily increased and at day 7 post-transfection eGFP expression again reached basal levels in all conditions. Interestingly, confocal images of cells transfected with NGs encapsulating labeled siRNA (siGLO green transfection indicator) revealed enlarged siGLO-NG containing vesicles in DL treated cells (Figure 7E). This indicates that not all lysosomally accumulated siRNA is released upon a single adjuvant treatment, which correlates well with previous reports [10,11]. Hence, we investigated if multiple DL treatments could induce additional siRNA release at later time points, using nuclease stabilized siRNA [19, 37]. We observed that upon multiple 2 hour DL treatments, minimal eGFP expression levels could be obtained up to 4 days post transfection, compared to negligible gene silencing in the absence of DL (Figure 7F). Despite the retained PLD phenotype by DL treatments after day 4 (Figure S7), the adjuvant treatments failed to provide further prolongation of the maximal gene silencing. This may be attributed to consecutive cell divisions that diluted the siNG-containing lysosomes to such an extent that minor additional siRNA release could no longer extend gene silencing. 
A
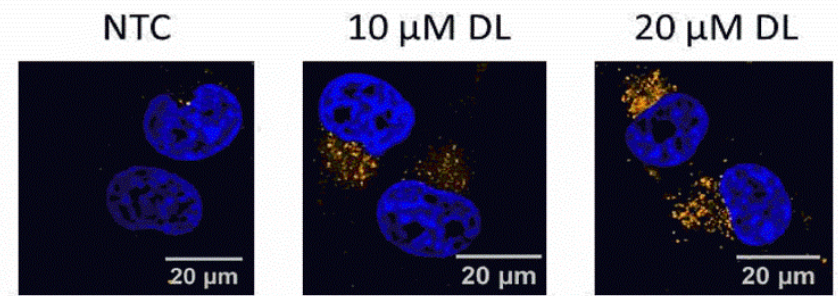

$40 \mu \mathrm{M} \mathrm{DL}$
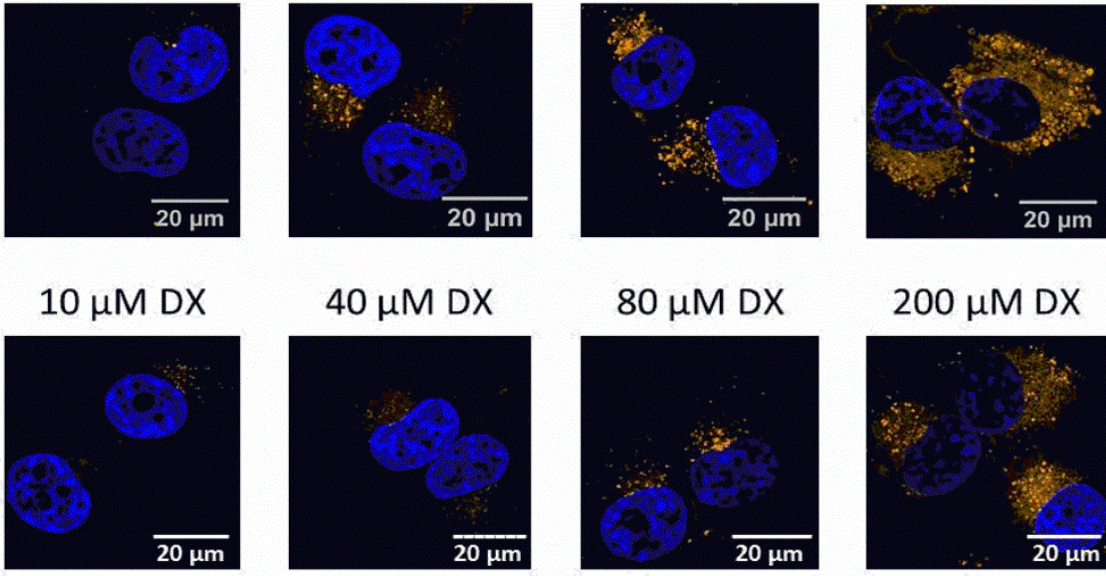

B

Untreated

$N G+40 \mu M D$

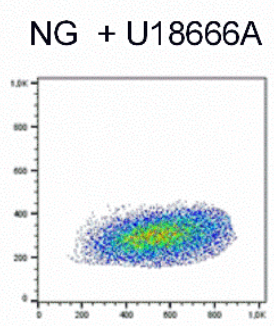

SSC
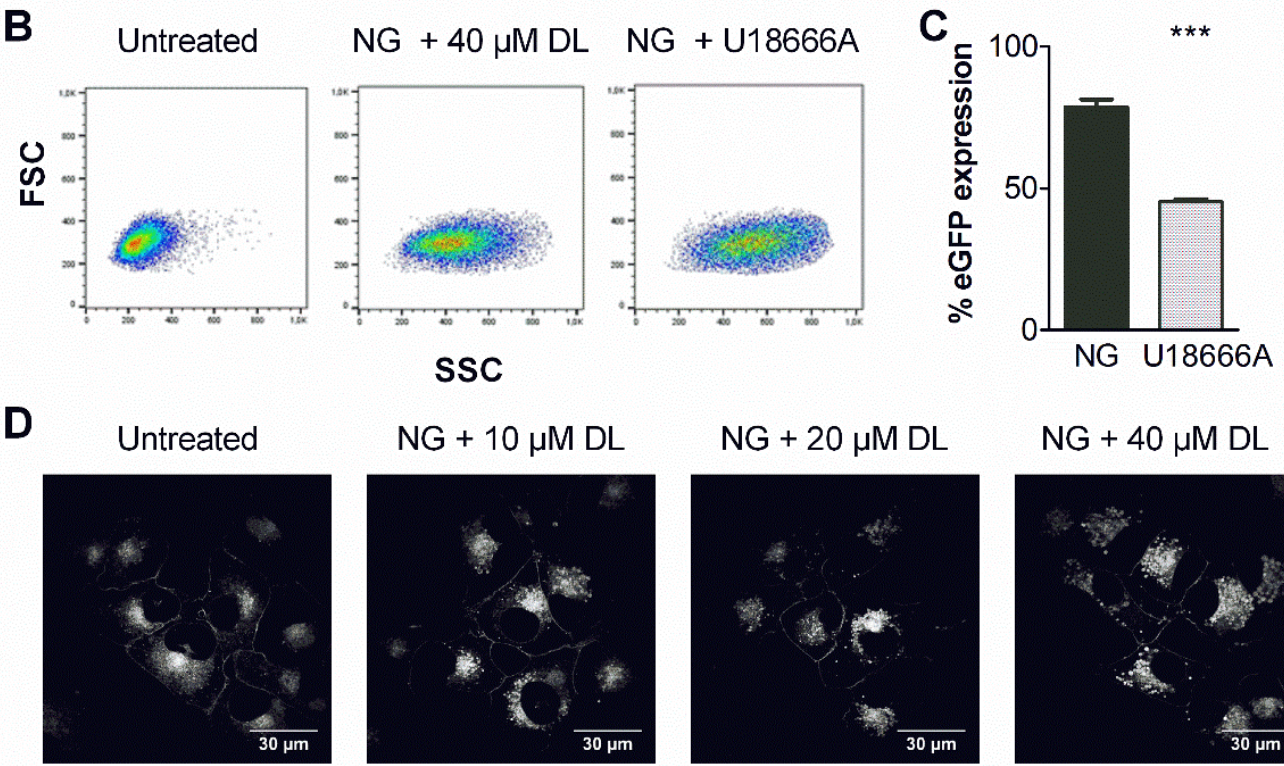

$\mathrm{NG}+10 \mu \mathrm{M} \mathrm{DL}$

$\mathrm{NG}+20 \mu \mathrm{M} \mathrm{DL}$

$200 \mu \mathrm{M} D X$
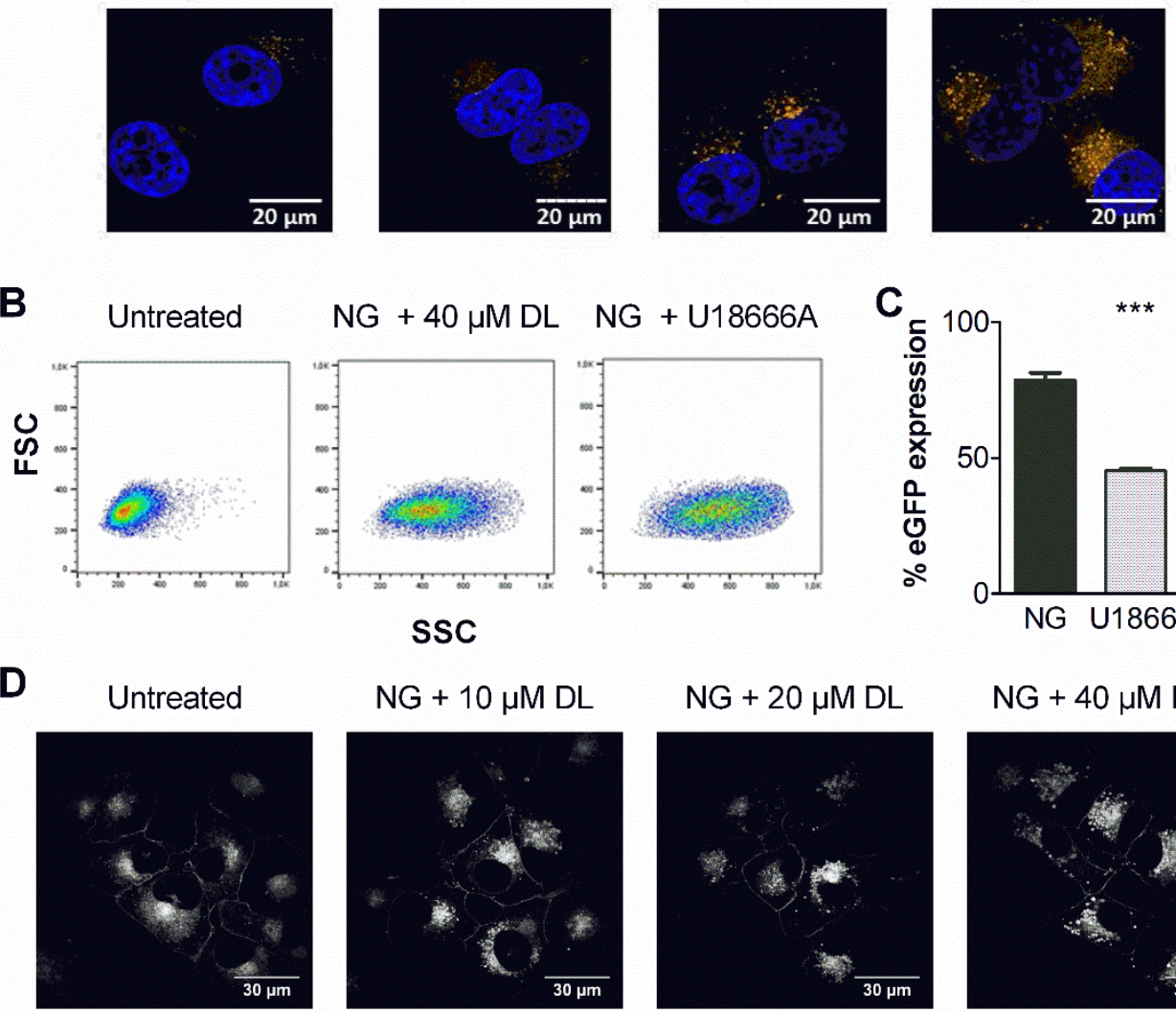

$\mathrm{NG}+40 \mu \mathrm{M} \mathrm{DL}$

E
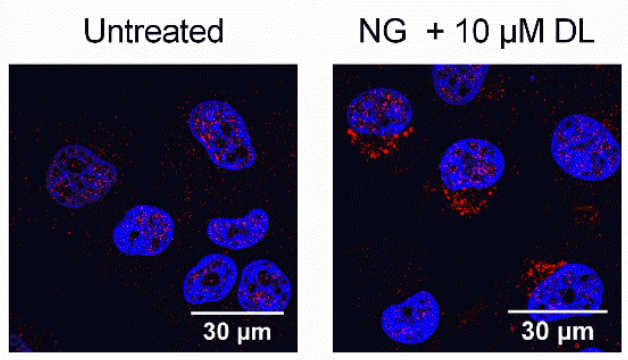

F

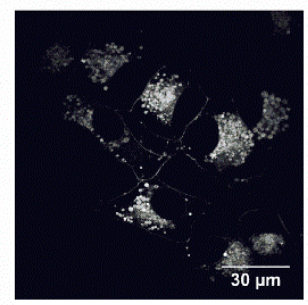

$\mathbf{F}$

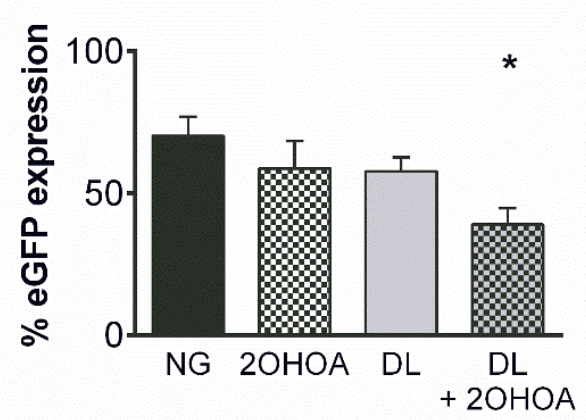

Figure 6. CADs induce a phospholipidosis phenotype. (A) Representative confocal images from the phospholipid distribution in H1299-WT cells visualized with LipidTOX ${ }^{\mathrm{TM}}$ Red PLD detection reagent in untreated and desloratadine $(D L)$ or dextrometorphan $(D X)$ treated cells. $(B)$

Representative scatter plots from untreated and siNG-transfected cells incubated with $40 \mu M D L$ or 30 $\mu M$ U18666A. (C) The eGFP silencing induced by adjuvant treatment with $30 \mu M$ U18666A compared 
to siNG transfection alone. (D) Intracellular cholesterol distribution in untreated H1299-WT cells or transfected cells treated with DL. (E) Intracellular SM distribution in untreated H1299-WT cells or transfected cells additionally treated with $10 \mu M D L$. (F) The percentage eGFP expression in H1299eGFP cells following transfection with the siNGs alone or in combination with $300 \mu \mathrm{M} 2 \mathrm{OHOA}, 10 \mu \mathrm{M}$ $D L$ or the combination of both. Data are represented as the mean \pm SEM $(n=3)$ and statistical significance is indicated when appropriate $\left({ }^{*} p<0.05,{ }^{* * *} p<0.005\right)$.

\subsection{Desloratadine improves therapeutic siRNA delivery}

Finally, we applied our adjuvant strategy to improve delivery of a therapeutic siRNA targeting polo-like kinase 1 (PLK1). Reducing PLK1 expression halts the cell cycle and induces apoptosis in p53 deficient cancer cells, such as the applied H1299 cell line [38, 39]. NGs loaded with control siRNA reduced the cell viability to approximately $80 \%$ in any condition tested, corroborating previous experiments (Figure S8A). The DL adjuvant treatment significantly enhanced siPLK1-mediated reduction in cell viability for all siPLK1 doses tested (Figure 7G, Figure S8B), with the combination of $40 \mu \mathrm{M}$ DL and $1 \mathrm{nM}$ siPLK1 reaching a comparable effect as $100 \mathrm{nM}$ siPLK1 without adjuvant support. Once again, repeating the adjuvant incubation (2 hours, $40 \mu \mathrm{M} \mathrm{DL}$ ) further reduced cell viability (Figure $7 \mathrm{H})$.

This approach requires confirmation in a relevant in vivo model, in which additional challenges like the carrier stability, the identification of suitable CAD doses and the selection of a sufficiently predictive in vivo model should be taken into account $[40,41]$. Importantly, it can be rationalized from the literature that this CAD adjuvant approach may likewise enhance siRNA delivery to cancer cells in vivo. Indeed, cancer cell lysosomes are generally less stable, show an altered membrane composition and lower ASM activity [14, 17, 42]. Of note, therapeutic doses of antidepressant CADs were shown to decrease ASM activity in vivo [18]. In addition, CADs were recently reported to induce LMP in cancer cells in vivo [14, 17]. Thus, we anticipate that sufficiently high adjuvant concentrations may be reached in tissues given the extremely high distribution volumes of CADs and, based on the data presented here, non-lethal LMP at lower CAD doses being sufficient to substantially promote siRNA delivery [43]. On the other hand, co-encapsulation of the delivery enhancing compound into the nanoparticle could further contribute to successful in vivo translation by conferring improved control over extra-and intracellular distribution, lowering the required dose or dosing frequency to observe an adjuvant effect and reducing systemic toxicity [44]. 
A

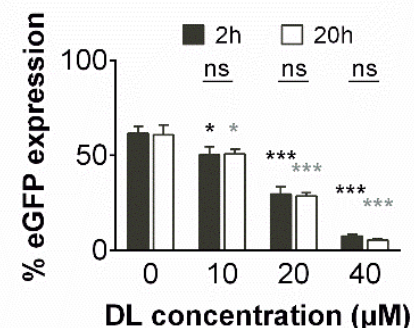

D

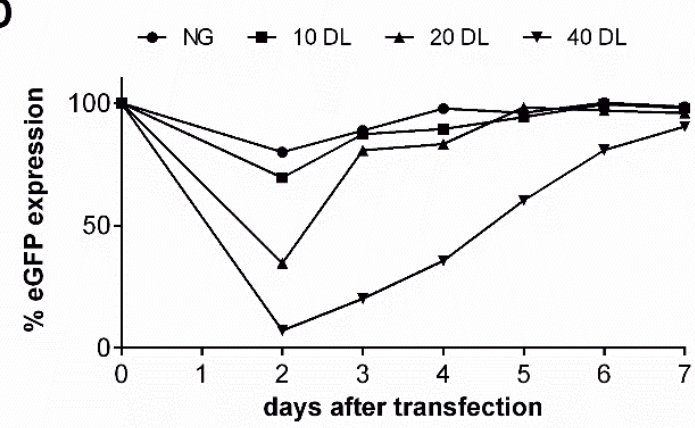

B

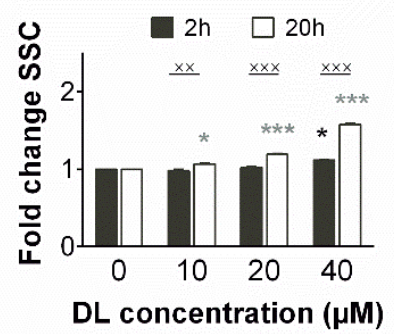

C

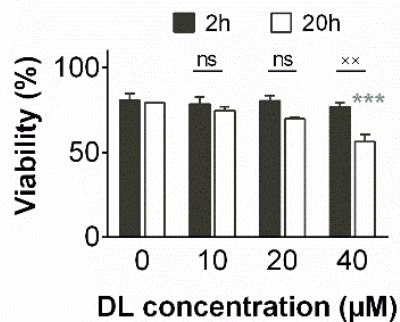

E

NG

$\mathrm{NG}+40 \mu \mathrm{M} \mathrm{DL}$
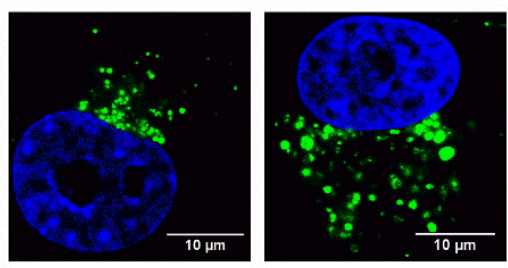

F

$\rightarrow \mathrm{NG} \rightarrow 1 \mathrm{xDL} \rightarrow \mathrm{DL}$ every other day $\rightarrow$ DL daily

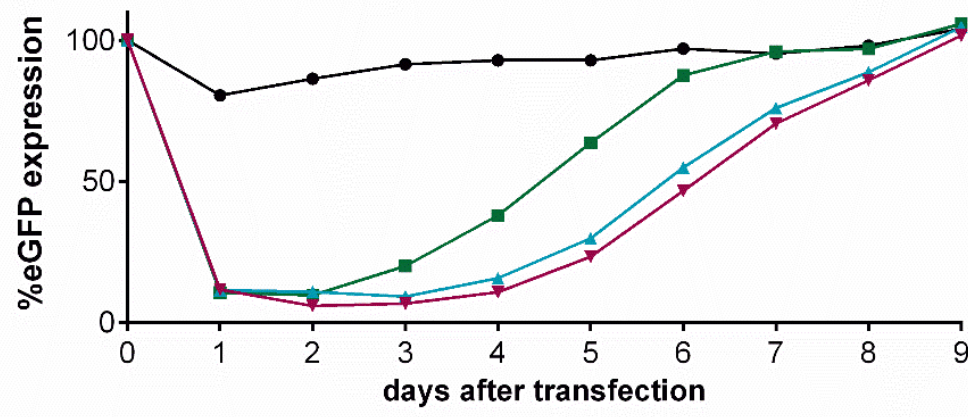

G

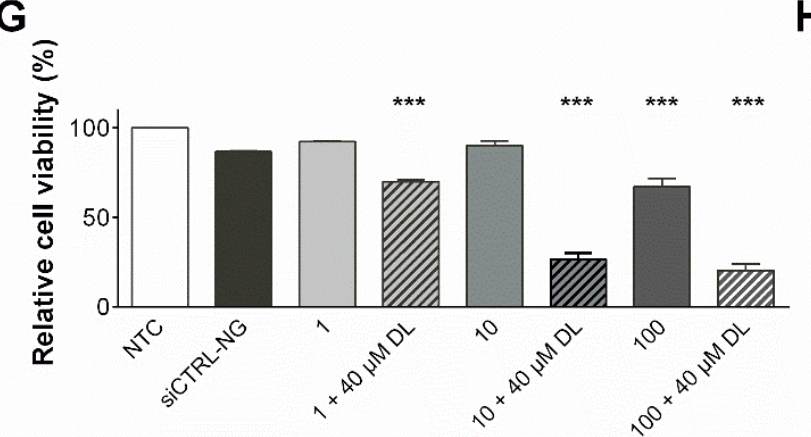

siPLK1 concentration (nM)
$\mathrm{H}$

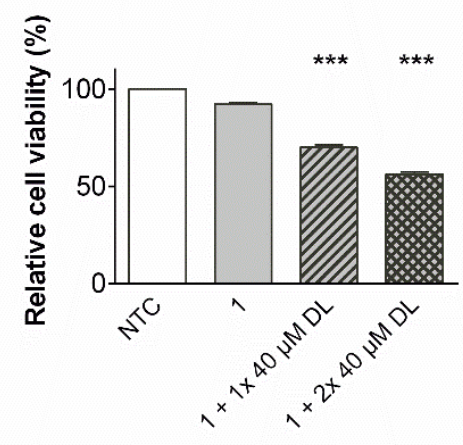

siPLK1 concentration ( $\mathrm{nM}$ )

Figure 7. The lysosomes as a depot for triggered siRNA release. (A-B) The percentage eGFP expression and fold change in side scatter (SSC) in H1299-eGFP cells transfected with siNGs alone or followed by a 2 hour or 20 hour desloratadine (DL) treatment. (C) Percentage viable cells following siNG transfection whether or not combined with 2 hour or 20 hour DL. (D) Kinetics of eGFP knockdown in siNG transfected cells followed by 2 hour DL incubation. (E) siGLO signal in H1299-WT cells transfected with siGLO-loaded NGs alone or followed by $40 \mu M D L$. (F) Kinetics of eGFP 
knockdown in cells transfected with siNGs and treated once with $40 \mu M D L$, daily or every other day until day six post transfection. (G) Percentage viable H1299-eGFP cells following transfection with siPLK1-loaded NGs whether or not subjected to a 2 hour sequential $40 \mu M D L$ treatment. (H) Percent viable cells after transfection with $1 \mathrm{nM}$ siPLK1-loaded NGs or subjected to one or two $40 \mu \mathrm{M} D L$ treatments. Data are represented as mean \pm SEM $(n=3)$ and statistical significance is indicated when appropriate by black * when referring to the untreated control and gray * when compared to NG transfected cells $\left({ }^{*} p<0.05,{ }^{* *} p<0.01,{ }^{* * *} p<0.005\right)$. Finally, statistical significance between the 2 hour and 20 hour condition is indicated by the black ${ }^{x}\left({ }^{x x} p<0.01,{ }^{x \times x} p<0.005\right)$. (NTC = untreated control, $n s$ $=$ not significant)

\section{CONCLUSION}

We demonstrated that cationic amphiphilic drugs (CADs) can be repurposed to stimulate functional lysosomal escape of siRNA in cancer cells. Indeed, CADs induced the accumulation of phospholipids, cholesterol and sphingomyelin, resulting in the enlargement of the lysosomal compartment, the induction of lysosomal membrane permeabilization (LMP) and enhanced cytosolic siRNA release. Importantly, our results strongly suggest that this acquired lysosomal storage disorder-phenotype and concurrent lysosomal destabilization are responsible for the improved siRNA delivery when CADs were sequentially applied to NG-transfected cancer cells. As the LMP phenotype is only transiently induced, it allows substantial siRNA delivery without affecting cell viability. Remarkably, multiple CAD treatments could trigger multiple rounds of siRNA release, highlighting the possibility to exploit lysosomes as vesicular depots for the cytosolic delivery of siRNA.

\section{ACKNOWLEDGMENTS}

FJ is a doctoral fellow of the Agency for Innovation by Science and Technology in Flanders (IWT). CB acknowledges the Education, Audiovisual and Culture Executive Agency (EACEA) of the European Commission in the field of the NanoFar Erasmus Mundus Joint Programme. LDB is a postdoctoral researcher of the Special Research Fund of Ghent University (BOF12/GOA/014). TVDV is a doctoral fellow of the Research Foundation-Flanders (FWO). KR also acknowledges the FWO for a postdoctoral Research Grant (grant 1517516N). 


\section{REFERENCES}

[1] A. Fire, S. Xu, M.K. Montgomery, S.A. Kostas, S.E. Driver, C.C. Mello, Potent and specific genetic interference by double-stranded RNA in Caenorhabditis elegans, Nature, 391 (1998) 806-811.

[2] K.V. Morris, S.W. Chan, S.E. Jacobsen, D.J. Looney, Small interfering RNA-induced transcriptional gene silencing in human cells, Science, 305 (2004) 1289-1292.

[3] B.L. Davidson, P.B. McCray, Jr., Current prospects for RNA interference-based therapies, Nat Rev Genet, 12 (2011) 329-340.

[4] A. Wittrup, J. Lieberman, Knocking down disease: a progress report on siRNA therapeutics, Nat Rev Genet, 16 (2015) 543-552.

[5] H. Yin, R.L. Kanasty, A.A. Eltoukhy, A.J. Vegas, J.R. Dorkin, D.G. Anderson, Non-viral vectors for gene-based therapy, Nat Rev Genet, 15 (2014) 541-555.

[6] T.F. Martens, K. Remaut, J. Demeester, S.C. De Smedt, K. Braeckmans, Intracellular delivery of nanomaterials: How to catch endosomal escape in the act, Nano Today, 9 (2014) 344-364.

[7] D. Ma, Enhancing endosomal escape for nanoparticle mediated siRNA delivery, Nanoscale, 6 (2014) 6415-6425.

[8] G. Sahay, W. Querbes, C. Alabi, A. Eltoukhy, S. Sarkar, C. Zurenko, E. Karagiannis, K. Love, D. Chen, R. Zoncu, Y. Buganim, A. Schroeder, R. Langer, D.G. Anderson, Efficiency of siRNA delivery by lipid nanoparticles is limited by endocytic recycling, Nat Biotechnol, 31 (2013) 653-658.

[9] S. Aits, M. Jaattela, Lysosomal cell death at a glance, J Cell Sci, 126 (2013) 1905-1912.

[10] A. Wittrup, A. Ai, X. Liu, P. Hamar, R. Trifonova, K. Charisse, M. Manoharan, T. Kirchhausen, J. Lieberman, Visualizing lipid-formulated siRNA release from endosomes and target gene knockdown, Nat Biotechnol, 33 (2015) 870-876.

[11] J. Gilleron, W. Querbes, A. Zeigerer, A. Borodovsky, G. Marsico, U. Schubert, K. Manygoats, S. Seifert, C. Andree, M. Stoter, H. Epstein-Barash, L.G. Zhang, V. Koteliansky, K. Fitzgerald, E. Fava, M. Bickle, Y. Kalaidzidis, A. Akinc, M. Maier, M. Zerial, Image-based analysis of lipid nanoparticlemediated siRNA delivery, intracellular trafficking and endosomal escape, Nature Biotechnology, 31 (2013) 638-U102.

[12] J. Kornhuber, M. Muehlbacher, S. Trapp, S. Pechmann, A. Friedl, M. Reichel, C. Muhle, L. Terfloth, T.W. Groemer, G.M. Spitzer, K.R. Liedl, E. Gulbins, P. Tripal, Identification of novel functional inhibitors of acid sphingomyelinase, PLoS One, 6 (2011) e23852.

[13] A.M. Villamil Giraldo, H. Appelqvist, T. Ederth, K. Ollinger, Lysosomotropic agents: impact on lysosomal membrane permeabilization and cell death, Biochem Soc Trans, 42 (2014) 1460-1464. [14] N.H. Petersen, O.D. Olsen, L. Groth-Pedersen, A.M. Ellegaard, M. Bilgin, S. Redmer, M.S. Ostenfeld, D. Ulanet, T.H. Dovmark, A. Lonborg, S.D. Vindelov, D. Hanahan, C. Arenz, C.S. Ejsing, T. Kirkegaard, M. Rohde, J. Nylandsted, M. Jaattela, Transformation-associated changes in sphingolipid metabolism sensitize cells to lysosomal cell death induced by inhibitors of acid sphingomyelinase, Cancer Cell, 24 (2013) 379-393.

[15] J. Kornhuber, P. Tripal, E. Gulbins, M. Muehlbacher, Functional inhibitors of acid sphingomyelinase (FIASMAs), Handb Exp Pharmacol, (2013) 169-186.

[16] P. Saftig, K. Sandhoff, Cancer: Killing from the inside, Nature, 502 (2013) 312-313.

[17] E. Gulbins, R.N. Kolesnick, It Takes a CAD to Kill a Tumor Cell with a LMP, Cancer Cell, 24 (2013) 279-281.

[18] E. Gulbins, M. Palmada, M. Reichel, A. Luth, C. Bohmer, D. Amato, C.P. Muller, C.H. Tischbirek, T.W. Groemer, G. Tabatabai, K.A. Becker, P. Tripal, S. Staedtler, T.F. Ackermann, J. van Brederode, C. Alzheimer, M. Weller, U.E. Lang, B. Kleuser, H. Grassme, J. Kornhuber, Acid sphingomyelinaseceramide system mediates effects of antidepressant drugs, Nat Med, 19 (2013) 934-938.

[19] K. Raemdonck, B. Naeye, K. Buyens, R.E. Vandenbroucke, A. Hogset, J. Demeester, S.C. De Smedt, Biodegradable Dextran Nanogels for RNA Interference: Focusing on Endosomal Escape and Intracellular siRNA Delivery, Adv. Funct. Mater., 19 (2009) 1406-1415. 
[20] S. Aits, M. Jaattela, J. Nylandsted, Methods for the quantification of lysosomal membrane permeabilization: A hallmark of lysosomal cell death, Method Cell Biol, 126 (2015) 261-285.

[21] W.H.t. Humphries, C.J. Szymanski, C.K. Payne, Endo-lysosomal vesicles positive for Rab7 and LAMP1 are terminal vesicles for the transport of dextran, PLoS One, 6 (2011) e26626.

[22] D.S. Wishart, C. Knox, A.C. Guo, S. Shrivastava, M. Hassanali, P. Stothard, Z. Chang, J. Woolsey, DrugBank: a comprehensive resource for in silico drug discovery and exploration, Nucleic Acids Res, 34 (2006) D668-672.

[23] L. De Backer, K. Braeckmans, J. Demeester, S.C. De Smedt, K. Raemdonck, The influence of natural pulmonary surfactant on the efficacy of siRNA-loaded dextran nanogels, Nanomedicine (Lond), 8 (2013) 1625-1638.

[24] R.S. Funk, J.P. Krise, Cationic amphiphilic drugs cause a marked expansion of apparent lysosomal volume: implications for an intracellular distribution-based drug interaction, Mol Pharm, 9 (2012) 1384-1395.

[25] X. Zhang, G.J. Sawyer, X. Dong, Y. Qiu, L. Collins, J.W. Fabre, The in vivo use of chloroquine to promote non-viral gene delivery to the liver via the portal vein and bile duct, J Gene Med, 5 (2003) 209-218.

[26] Z.U. Rehman, D. Hoekstra, I.S. Zuhorn, Mechanism of Polyplex- and Lipoplex-Mediated Delivery of Nucleic Acids: Real-Time Visualization of Transient Membrane Destabilization without Endosomal Lysis, ACS Nano, 7 (2013) 3767-3777.

[27] R. Logan, A.C. Kong, E. Axcell, J.P. Krise, Amine-containing molecules and the induction of an expanded lysosomal volume phenotype: a structure-activity relationship study, J Pharm Sci, 103 (2014) 1572-1580.

[28] W.H. Halliwell, Cationic amphiphilic drug-induced phospholipidosis, Toxicol Pathol, 25 (1997) 53-60.

[29] D. te Vruchte, A.O. Speak, K.L. Wallom, N. Al Eisa, D.A. Smith, C.J. Hendriksz, L. Simmons, R.H. Lachmann, A. Cousins, R. Hartung, E. Mengel, H. Runz, M. Beck, Y. Amraoui, J. Imrie, E. Jacklin, K. Riddick, N.M. Yanjanin, C.A. Wassif, A. Rolfs, F. Rimmele, N. Wright, C. Taylor, U. Ramaswami, T.M. Cox, C. Hastings, X.T. Jiang, R. Sidhu, D.S. Ory, B. Arias, M. Jeyakumar, D.J. Sillence, J.E. Wraith, F.D. Porter, M. Cortina-Borja, F.M. Platt, Relative acidic compartment volume as a lysosomal storage disorder-associated biomarker, J Clin Invest, 124 (2014) 1320-1328.

[30] C.J. Shoemaker, K.L. Schornberg, S.E. Delos, C. Scully, H. Pajouhesh, G.G. Olinger, L.M. Johansen, J.M. White, Multiple Cationic Amphiphiles Induce a Niemann-Pick C Phenotype and Inhibit Ebola Virus Entry and Infection, Plos One, 8 (2013).

[31] T. Kirkegaard, A.G. Roth, N.H. Petersen, A.K. Mahalka, O.D. Olsen, I. Moilanen, A. Zylicz, J. Knudsen, K. Sandhoff, C. Arenz, P.K. Kinnunen, J. Nylandsted, M. Jaattela, Hsp70 stabilizes lysosomes and reverts Niemann-Pick disease-associated lysosomal pathology, Nature, 463 (2010) 549-553.

[32] G. Barcelo-Coblijn, M.L. Martin, R.F. de Almeida, M.A. Noguera-Salva, A. Marcilla-Etxenike, F. Guardiola-Serrano, A. Luth, B. Kleuser, J.E. Halver, P.V. Escriba, Sphingomyelin and sphingomyelin synthase (SMS) in the malignant transformation of glioma cells and in 2-hydroxyoleic acid therapy, Proc Natl Acad Sci U S A, 108 (2011) 19569-19574.

[33] H. Wang, Y.Y. Tam, S. Chen, J. Zaifman, R. van der Meel, M.A. Ciufolini, P.R. Cullis, The NiemannPick C1 Inhibitor NP3.47 Enhances Gene Silencing Potency of Lipid Nanoparticles Containing siRNA, Mol. Ther., 24 (2016) 2100-2108.

[34] U. Repnik, M.B. Distefano, M.T. Speth, M.Y.W. Ng, C. Progida, B. Hoflack, J. Gruenberg, G. Griffiths, LLOMe does not release cysteine cathepsins to the cytosol but inactivates them in transiently permeabilized lysosomes, J. Cell Sci., 130 (2017) 3124-3140.

[35] R. Gomez-Sintes, M.D. Ledesma, P. Boya, Lysosomal cell death mechanisms in aging, Ageing research reviews, 32 (2016) 150-168.

[36] V. Stoka, V. Turk, B. Turk, Lysosomal cathepsins and their regulation in aging and neurodegeneration, Ageing research reviews, 32 (2016) 22-37. 
[37] K. Raemdonck, B. Naeye, A. Hogset, J. Demeester, S.C. De Smedt, Prolonged gene silencing by combining siRNA nanogels and photochemical internalization, J Control Release, 145 (2010) 281-288. [38] E. Kawata, E. Ashihara, S. Kimura, K. Takenaka, K. Sato, R. Tanaka, A. Yokota, Y. Kamitsuji, M. Takeuchi, J. Kuroda, F. Tanaka, T. Yoshikawa, T. Maekawa, Administration of PLK-1 small interfering RNA with atelocollagen prevents the growth of liver metastases of lung cancer, Molecular Cancer Therapeutics, 7 (2008) 2904-2912.

[39] J.A. McCarroll, T. Dwarte, H. Baigude, J. Dang, L. Yang, R.B. Erlich, K. Kimpton, J. Teo, S.M. Sagnella, M.C. Akerfeldt, J. Liu, P.A. Phillips, T.M. Rana, M. Kavallaris, Therapeutic targeting of pololike kinase 1 using RNA-interfering nanoparticles (iNOPs) for the treatment of non-small cell lung cancer, Oncotarget, 6 (2015) 12020-12034.

[40] B. Naeye, H. Deschout, M. Roding, M. Rudemo, J. Delanghe, K. Devreese, J. Demeester, K. Braeckmans, S.C. De Smedt, K. Raemdonck, Hemocompatibility of siRNA loaded dextran nanogels, Biomaterials, 32 (2011) 9120-9127.

[41] S.E. Gould, M.R. Junttila, F.J. de Sauvage, Translational value of mouse models in oncology drug development, Nat Med, 21 (2015) 431-439.

[42] P. Boya, G. Kroemer, Lysosomal membrane permeabilization in cell death, Oncogene, 27 (2008) 6434-6451.

[43] A.M. Ellegaard, C. Dehlendorff, A.C. Vind, A. Anand, L. Cederkvist, N.H.T. Petersen, J. Nylandsted, J. Stenvang, A. Mellemgaard, K. Osterlind, S. Friis, M. Jaattela, Repurposing Cationic Amphiphilic Antihistamines for Cancer Treatment, Ebiomedicine, 9 (2016) 130-139.

[44] F. Joris, S.C. De Smedt, K. Raemdonck, Small molecules convey big messages: Boosting non-viral nucleic acid delivery with low molecular weight drugs, Nano Today, (2017)

doi.org/10.1016/j.nantod.2017.06.012 